\title{
Regulation mechanism of miR-494-3p on endometrial receptivity in mice via $\mathrm{PI} 3 \mathrm{~K} / \mathrm{AKT} / \mathrm{mTOR}$ pathway
}

\author{
Lan Yuan ${ }^{1, \star}$, Fen Feng ${ }^{2, *}$, Zhu Mao ${ }^{3}$, Jin-zhu Huang ${ }^{3}$, Yi Liu' ${ }^{1}$, Yu-lin Li ${ }^{1}$ and Rong-xing Jiang ${ }^{2}$ \\ ${ }^{1}$ School of Medical and Life Sciences/Reproductive and Women-Children Hospital, Chengdu University of Traditional \\ Chinese Medicine, Chengdu, Sichuan, P.R. China \\ ${ }^{2}$ Hospital of Chengdu University of Traditional Chinese Medicine, Chengdu, Sichuan, P.R. China \\ ${ }^{3}$ School of Nursing, Chengdu University of Traditional Chinese Medicine, Chengdu, Sichuan, P.R. China
}

\begin{abstract}
Successful implantation requires endometrial receptivity. To investigate the mechanisms of miR-494-3p on endometrial receptivity, GnRHa's superovulation scheme was designed to reduce endometrial receptivity, and the pregnant mice were injected with miR-494-3p antagomir. The regulatory role of miR-494-3p was identified by RT-qPCR, uterine blastocyst count, scanning electron microscopy, hematoxylin-eosin (HE) staining, and Western blot. Results indicated that miR-494-3p antagomir increased uterine blastocysts numbers, promoted the pinocytosis expressions, and increased endometrial thickness. Besides, miR-494-3p antagomir significantly increased leukemia inhibitory factor (LIF), Ang-2 and VEGF protein expressions, and up-regulated p-AKT/AKT and p-mTOR/mTOR protein ratios in endometrium. Luciferase assay confirmed that LIF was a potential target of miR-494-3p. Subsequently, human endometrial epithelial cells (hEECs) were transfected with miR-494-3p inhibitor and PI3K inhibitor (LY294002). The role of miR-494-3p was identified by RT-qPCR, CCK-8 assay, transwell assay and flow cytometry. Results indicated that miR-494-3p inhibitor significantly increased proliferation and invasion, and significantly inhibited apoptosis in hEECs, while LY294002 reversed its biological function. Overall, these results suggested that miR-494-3p is the key regulator of endometrial receptivity in mice, regulating this complex process through the $\mathrm{PI} 3 \mathrm{~K} / \mathrm{AKT} / \mathrm{mTOR}$ pathway. Understanding the role of miR-494-3p in endometrial receptivity is of great significance for exploring new targets for the diagnosis and treatment of early pregnancy failure, and improving the success rates of artificial reproduction.
\end{abstract}

Key words: Endometrial receptivity - miR-494-3p - PI3K/AKT/mTOR pathway - Leukemia inhibitory factor

\section{Introduction}

At present, repeated implantation failure of embryos is a serious challenge to assisted reproduction technology, and endometrial receptivity plays a key role in the process of embryo implantation (Mahajan et al. 2015). Only when the endometrium has good receptivity can the blastocyst

\footnotetext{
* These authors contributed equally to this work.

Correspondence to: Rong-xing Jiang, No. 39-41, Ziqiao Road, Jinniu District, Chengdu, Sichuan, P.R. China

E-mail: jiangrx@cdutcm.edu.cn
}

be accepted and accommodated to complete the process of localization, adhesion and implantation (Guo et al. 2018). If the endometrial receptivity is too low to reach the required conditions for embryo implantation, the embryo transfer rate will be greatly reduced, so that the implantation process cannot be completed, and the endometrial morphology and related factors will change (Chuffa et al. 2019). Some of these factors have been confirmed to be involved in the regulation of endometrial receptivity (Kunickaet al. 2020). Therefore, the study of markers of endometrial receptivity can not only monitor the change of endometrial receptivity, but also improve endometrial receptivity by influencing

(c) The Authors 2021. This is an open access article under the terms of the Creative Commons Attribution-NonCommercial 4.0 International License (https://creativecommons.org/licenses/by-nc/4.0/), which permits non-commercial use, distribution, and reproduction in any medium, provided the original work is properly cited. 
the expression of some markers, which may be the key to improve the success of embryo transplantation.

Studies have shown that gonadotropin releasing hormone agonists (GnRHA) assisted superovulation can effectively prevent the generation of ultra-high level luteinizing hormone (LH) and its peak in the follicular stage and prevent premature luteinization of follicles. It is beneficial to improve the quality of ovum and the environment of endometrial implantation. It has no significant effect on the membrane thickness in the proliferative stage or even the early secretory stage, and is significantly better than urinary gonadotropin (HMG) superovulation regimen alone, which can improve the success rate of in vitro fertilization-embryo transfer (IVF-ET) (Nikas et al. 1999).

Leukemia inhibitory factor (LIF) is induced by estrogen and is necessary for embryo implantation and endometrial stromal cell decidualization. A large number of studies have shown that LIF plays a certain role in embryo implantation and embryo development (Franasiak et al. 2014). Downregulated LIF expression is associated with implantation defects in female infertility. In many mammals, including humans, LIF expression is significantly increased during implantation, indicating that LIF is a key factor in the regulation of implantation (Schmitz et al. 2017). Besides, angiopoietin-2 (Ang-2) is involved in villi formation during implantation and the establishment of fetal and maternal capillary network, which is very important for maintaining pregnancy (Fiorimanti et al. 2018). In addition, endometrial angiogenesis plays an important role in the evaluation of endometrial receptivity. Angiogenesis refers to the growth of new blood vessels on the basis of the original vascular bed, which exists in the process of tissue growth, development and repair, and is closely related to tumor growth (Huang et al. 2020). Therefore, the regulation of angiogenesis has important clinical application value. Vascular endothelial growth factor (VEGF), as an endothelial-specific mitogen in vitro, is known to be a key factor in angiogenesis and angiogenesis in various models (Chen et al. 2020; Li et al. 2020). In rodents, VEGF plays a role in embryonic-endometrial interaction by regulating endometrial vascular permeability and endothelial cell proliferation at the implantation site (Kaczmarek et al. 2010; Złotkowska et al. 2019). Thus, these factors may be involved in endometrial receptivity.

MicroRNA (miRNA) is a small endogenous non-coding RNA molecule composed of about 22 nucleotide sequences. These small miRNAs usually target one or more mRNAs and regulate gene expression by inhibiting or blocking target mRNAs at the translation level (Griffiths-Jones et al. 2006). In recent years, a large number of studies have found that miRNA is closely related to endometrial receptivity. Studies have shown that miRNAs can affect the expression of intima receptivity marker LIF in the endometrial tissue (Zhang et al. 2019). The differential expression of miRNA leads to syn- chronous changes of related target genes, which in turn leads to a series of changes in the regulatory pathway and network system of endometrium, thus establishing an endometrial state most suitable for blastocyst implantation and ensuring the smooth progress of pregnancy (Hosseini et al. 2020; Kim et al. 2020). Therefore, in-depth study of miRNA and its regulated target genes is the most important and direct way to understand the establishment and maintenance of endometrial receptance, which will play an important role in understanding and evaluating endometrial function, and predicting pregnancy outcome. Recently, Altmäe et al. (2013) compared the expression changes of miRNA in endometrial samples from healthy women of reproductive age in the early and middle stages of secretion, and found that the expression of miR-494 was significantly downregulated in endometrial samples from women in metaphase secretion. Another study found that miR-494-3p was related to endometrial receptivity in the microRNA expression profiling of endometriaassociated infertility patients (Xu et al. 2017). Furthermore, overexpression of miR-494-3p has been shown in previous studies to block the activity of $\mathrm{PI} 3 \mathrm{~K} / \mathrm{AKT} / \mathrm{mTOR}$ pathway (Yuan et al. 2020). However, up to now, there has been no study on the relevant mechanism of how miR-494-3p affects endometrial receptivity via the $\mathrm{PI} 3 \mathrm{~K} / \mathrm{AKT} / \mathrm{mTOR}$ pathway, so its mechanism of action cannot be clearly defined.

In the process of conception, high quality embryo and good endometrial receptivity are key to embryo implantation. How to improve endometrial receptivity to increase the rate of embryo implantation and pregnancy is the key issue we are committed to studying. The purpose of this study was to investigate the effect of miR-494-3p targeting LIF via $\mathrm{PI} 3 \mathrm{~K} / \mathrm{AKT} / \mathrm{mTOR}$ pathway on endometrial receptivity regulation in mice.

\section{Materials and Methods}

\section{Reagents and animals}

Alarelin (GnRHa) was purchased from Maanshan Fengyuan Pharmaceutical Co. LTD (SFDA approval number: H20041093; batch number: 1005265; China). Urinary gonadotropin (HMG) (SFDA approval number: H10940097; batch number: 1007234; China) and Chorionic gonadotropin (HCG) (SFDA approval number: H44020674; batch number: 1006137; China) were purchased from Lizhu Group Libao Biochemical Pharmaceutical Co. LTD. Gibco Dulbecco's Modified Eagle's Medium with low glucose (LG-DMEM) and fetal bovine serum (FBS) were purchased from ThermoFisherScientific (Waltham, MA). Trizol reagent was purchased from Invitrogen (Carlsbad, CA, USA). MirVana ${ }^{\mathrm{TM}}$ miRNA Isolation Kit, Mir-X miRNA First-Strand Synthesis kit (Takara Bio, Inc.), Mir-X miRNA qRT-PCR TB Green ${ }^{\circledR}$ 
Kit, miRNA primer, Opti-MEM ${ }^{\circledR}$, Lipofectamine ${ }^{\circledR}$ 2000, miR-494-3p antagomir, NC-antagomir, miR-494-3p mimic negative control (mimic-NC), miR-494-3p mimic, miR-494$3 p$ inhibitor NC (NC-inhibitor), miR-494-3p inhibitor were purchased from GenePharma (Suzhou, China). The LIF, Ang-2, VEGF, AKT, p-AKT, mTOR and p-mTOR antibodies were purchased from Abcam. SPF Grade C57BL6 mice were purchased from Experimental Animal Center West China Hospital Sichuan University.

\section{Animal study}

Forty non-pregnant female mice of SPF Grade C57BL6 and 20 healthy male mice of the same breed were selected, aged 6-8 weeks and weighing 25-30 g. After two days of adaptive feeding, the mice were given vaginal exfoliated cell smears every day for two weeks. Twenty mice with normal estrous cycle were selected for the test, and were randomly divided into four groups: NC (negative control) group, GnRHa group (injecting GnRHa), GnRHa+NC-antagomir group (injecting GnRHa and NC-antagomir), and GnRHa+miR-494-3p antagomir group (injecting GnRHa and miR-494-3p antagomir). Specifically, except for the NC group, mice in the other groups were injected intraperitoneally with $40 \mu \mathrm{g} / 100 \mathrm{~g}$ of GnRHa to induce implantation disorder (Gong et al. 2013; Yuan et al. 2020) at 9:00 a.m. every day for 9 consecutive days. At the 9th day, the mice were injected with $0.04 \mathrm{IU} /$ $\mathrm{kg}$ HMG for ovulation induction, and $0.1 \mathrm{IU} / \mathrm{kg}$ HCG was injected $48 \mathrm{~h}$ later. At 6:00 p.m. on that day, the mice were caged in a ratio of $2: 1$ female to male. Female mice were observed on the next day, and the day of vaginal blockage was regarded as the first day of pregnancy.

To examine the influence of miR-494-3p on embryo implantation, mice in the GnRHa group, GnRHa+NC-antagomir group or GnRHa+miR-494-3p antagomir group were injected with RNase-free water, NC-antagomir or miR-494$3 \mathrm{p}$ antagomir through the tail vein after $48 \mathrm{~h}$ of pregnancy, respectively, on day 3 at 9:00 a.m. Sequences were 5'-GAGGUUUCCCGUGUAUGUUUCA-3' (miR-494-3p antagomir; Suzhou, China) and 5'-CAGUACUUUUGUGUAGUACAA-3' (NC-antagomir; Suzhou, China). Each mouse was injected with $0.3 \mathrm{ml} 5 \%$ Trypan Blue intravenously on 5th day after pregnancy. After $10 \mathrm{~min}$, the mice were sacrificed by cervical dislocation. The mice were then laparotomized. The embryo implantation sites of test mice were detected and the pregnancy rate and implantation blastocysts of biangular uterus were counted. The separation of endometrium was as follows: the endometrium was removed in a sterile environment, and the mesangial and adipose tissue were removed. Ophthalmic tweezers were used to clamp the unilateral uterine horn, and the treated 4-point needle was used to squeeze and roll gradually from the near end of the fallopian tube to the cervix, so that the endometrium presented a long strip and slid out completely. Then, liquid nitrogen was quickly placed into a clean EP tube for preservation. One side of the bi-corner uterus was fixed in $4 \%$ paraformaldehyde for hematoxylin-eosin (HE) staining observation. The other side of the bi-corner uterus was longitudinally opened, washed with normal saline, gently flattened and fixed in 3\% glutaraldehyde for scanning electron microscope observation.

\section{Scanning electron microscopy observation}

After fixation, the specimens were thoroughly washed with phosphate buffer and fixed with $2.5 \%$ glutaraldehyde and $1 \%$ osmium for $2 \mathrm{~h}$, followed by gradient dehydration with ethanol. Then, the samples were freeze-dried in a freeze-drying apparatus, pasted and sprayed with gold in an ion sputtering apparatus for $3 \mathrm{~min}$. The pinopodes were observed under a JSM-820 scanning electron microscope.

\section{$R N A$ extraction and $R T-q P C R$}

Total RNA from endometrial tissues and human endometrial epithelial cells (hEECs) were extracted using Trizol reagent in accordance with the manufacturer's instructions, respectively. $5 \mu \mathrm{g}$ of total RNA was taken and synthesized into cDNA by reverse transcription reaction. Purified RNA was used as a template to synthesize cDNA using Mir-X miRNA First-Strand Synthesis kit. RT PCR was run with cDNA, Mir-X miRNA qRT-PCR TB Green ${ }^{\circledR}$ Kit and gene specific primers for miR-494-3p. All primers were designed by Sangon Biotech (Shanghai) Co., Ltd. The primer sequences for miR-494-3p: Forward: 5'-TGAAACATACACGGGAAACCTC-3', Reverse: Mir-X miRNA qRT-PCR TB Green ${ }^{\circledR}$ Kit provided. The primer sequences for U6 were provided by Mir-X miRNA qRT-PCR TB Green ${ }^{\circledR}$ Kit. The reaction conditions were as follows: 1 cycle at $95^{\circ} \mathrm{C}$ for $10 \mathrm{~s}$ followed by 40 cycles at $95^{\circ} \mathrm{C}$ for $5 \mathrm{~s}$, and $60^{\circ} \mathrm{C}$ for $20 \mathrm{~s}$. After the reaction, the $\mathrm{Ct}$ value of each sample was automatically analyzed by the computer, and the relative mRNA expression was calculated by $2^{-\Delta \Delta \mathrm{Ct}}$ with $\mathrm{U} 6$ as the internal control. The specificity of PCR reaction was determined by the melting curve.

\section{Histological examination}

Paraffin-embedded uterine tissue was sectioned into $4 \mu \mathrm{m}$ thick fragments using a paraffin microtome. Two sections of each mouse uterus were randomly selected for HE staining, and each section was photographed under high magnification $(100 \times)$ and low magnification $(40 \times)$. Four fields were randomly selected, and the endometrial thickness, namely the vertical distance between the intimal myometrium junction and the uterine cavity, was determined by Leica Qwin Plus image processing software, and the mean value was taken as the endometrial thickness of this section. 


\section{Western blot analysis}

The preserved endometrial tissue was removed. The intima was quickly scraped on the ice and collected to a $1.5 \mathrm{ml}$ EP tube. Precooled RIPA lysis buffer of $200 \mu \mathrm{l}$ was added and reacted on ice for $30 \mathrm{~min}$. Then, ultrasonic was used to grind it for 3 times, $5 \mathrm{~s}$ each time. Centrifugation was performed at $4^{\circ} \mathrm{C}$ and $12,000 \mathrm{r} / \mathrm{min}$ for $15 \mathrm{~min}$. The supernatant was boiled at $100^{\circ} \mathrm{C}$ for $10 \mathrm{~min}$ and then the protein was quantified. After sample loading, $10 \%$ polyacrylamide was subjected to SDS-PAGE electrophoresis and transferred to cellulose nitrate film. 5\% BSA blocking solution was shaken and incubated for $1 \mathrm{~h}$ at room temperature. The primary antibodies were added in a proportional dilution: anti-LIF (ab113262; 1:1000, Abcam), anti-Ang-2 (ab153934; 1:1000, Abcam), anti-VEGF (ab32152; 1:2000, Abcam), AKT (ab8805; 1:1,000, Abcam), p-AKT (ab38449; 1:1,000, Abcam), mTOR (ab2732; 1:2,000, Abcam) and p-mTOR (ab109268; 1:1,000, Abcam). After incubation at $4^{\circ} \mathrm{C}$ overnight, TBST membrane was washed for $10 \mathrm{~min} 3$ times. Then the corresponding secondary antibody (1:400) was added and incubated at room temperature for $1 \mathrm{~h}$. The TBST membrane was washed for 15 min 3 times, and the chemiluminescence reaction was performed for development. Image-J image analysis software was used to analyze the expression level of the target protein, and the gray value ratio of the target protein stripe to the $\beta$-actin stripe of the same swimlane was taken as the measurement results for comparative analysis.

\section{Cell culture and treatment}

hEECs (Yanyu biotechnology, Shanghai, China) were cultured in DMEM (Gibco, Suzhou, China) with 7\% FBS (Gibco, Suzhou, China) and antibiotics (1\% penicillin/ streptomycin; Solarbio, Beijing, China) at $37^{\circ} \mathrm{C}$ in a humidified air containing $5 \% \mathrm{CO}_{2}$ incubator. Logarithmic growth hEECs were digested with trypsin and resuspended in a medium containing $10 \%$ FBS. The cells $\left(3 \times 10^{5}\right)$ were then inoculated in a 6-well culture plate and transfected with either miR-494-3p mimic negative control (mimic-NC), miR-494-3p mimic, miR-494-3p inhibitor NC (NC-inhibitor), miR-494-3p inhibitor, or PI3K inhibitor (LY294002) using Lipofectamine 2000 (Invitrogen) according to the manufacturer's instructions. Briefly, when cultured cells in the incubator were fused to 50\%, opti-MEM medium and LipofectamineTM 2000 were gently mixed with each sequence and incubated separately for $5 \mathrm{~min}$. The mixture was then mixed and incubated at room temperature for 20 min. The mixture was evenly added to the culture medium and placed in an incubator. After waiting for $6 \mathrm{~h}$, the original culture medium was replaced with a fresh anti-double culture medium containing 10\% FBS.

\section{Luciferase assay}

In order to determine the regulatory relationship between miR-494-3p and LIF, a luciferase assay was carried out in this study using specifically designed luciferase vectors. In brief, the full 3'UTR sequences of LIF containing the binding sites for miR-494-3p were amplified and subcloned into pcDNA vectors (Promega) to generate wild-type vectors for LIF. Then, hEEC cells were seeded into 96-well plates and co-transfected with miRNA-34c mimics/mimic-NC (5 pmol) and LIF-3'UTR-WT (0.16 ug) using Lipofectamine 2000. Finally, luciferase activity of transfected cells was assayed after $48 \mathrm{~h}$ of transfection using a Dual-Luciferase reporter assay system (Promega, Madison, WI, USA).

\section{CCK-8 assay}

The hEEC cells were inoculated in 96-well plates and transfected the next day. Cells not transfected with any plasmids, transfected with miR-494-3p inhibitor, or co-transfected with miR-494-3p inhibitor and PI3K inhibitor, were added with the CCK-8 reagent in each well after $48 \mathrm{~h}$ of transfection. Absorbance (A) at $450 \mathrm{~nm}$ was recorded in accordance with the Cell Counting Kit-8 (CCK-8; Dojindo Laboratories, Kumamoto, Japan). Finally, the survival curve was drawn according to the cell proliferation.

\section{Flow cytometry analysis}

The semi-fused hEEC cells were transfected with miR-494$3 p$ inhibitor or none plasmid, co-transfected with miR-494$3 \mathrm{p}$ inhibitor and PI3K inhibitor, respectively. After $48 \mathrm{~h}$, all cells were collected and stained with Annexin V-FITC (MultiSciences) and propidium iodide (PI). Cell apoptosis was determined by flow cytometry using annexin $\mathrm{V}$ and PI double-staining technique for phosphatidylserine membrane redistribution. Then the number of apoptotic cells was measured by BD Caliber flow cytometry (Becton Dickinson).

\section{Cell invasion assays}

Transwell assays were performed at $24 \mathrm{~h}$ after transfection. Briefly, hEEC cell suspension of $100 \mu \mathrm{l} /$ cell was inoculated into transwell supraventricular chamber to achieve a final concentration of $8 \times 10^{4}$ cells/chamber, with Matrigel Matrix coating solution from Corning (Corning, NY, USA) for the assay. Then, $750 \mu \mathrm{l}$ serum-containing medium was added to the lower chamber. Finally, after $48 \mathrm{~h}$ of culture, transwell chamber was taken out and stained with crystal violet. Photographs were taken randomly under the microscope to collect experimental data for analysis. 


\section{Statistical analysis}

Data were expressed as mean \pm standard deviation. SPSS 17.0 (SPSS Inc.) was used to conduct one-way analysis of variance with Tukey's multiple comparison test for the differences between groups. $p<0.05$ was considered statistically significant.

\section{Results}

Inhibition of miR-494-3p improved endometrial receptivity

After injecting miR-494-3p antagomir, NC-antagomir or RNase-free water, the miR-494-3p expressions of endometrial epithelium cells (EECs) in mice were determined by RT-qPCR. Compared with the NC group, the expression of miR-494-3p was significantly increased in both the GnRHa group and the GnRHa+NC-antagomir group. While when EECs of GnRHa superovulation mice were stimulated with miR-494-3p-antagomir, no significant difference in GnRHa+miR-494-3p-antagomir group was detected. Compared with the GnRHa group, the expression of miR-494-3p was significantly decreased in the GnRHa+miR-494-3pantagomir group, while the $\mathrm{GnRHa}+\mathrm{NC}$-antagomir group showed no significant difference (Fig. 1A).

To investigate the effect of miR-494-3p on endometrial receptivity, we observed the expression of pinopodes under a scanning electron microscope and counted the number of intrauterine blastocysts. In the NC group, the endometrial surface was distributed with many clusters of membranelike protuberances, namely the developing pinopodes. The size and shape were similar, and the boundary between the membranous bulges was obvious. In the GnRHa group and GnRHa+NC-antagomir group, the developments of pino-
A

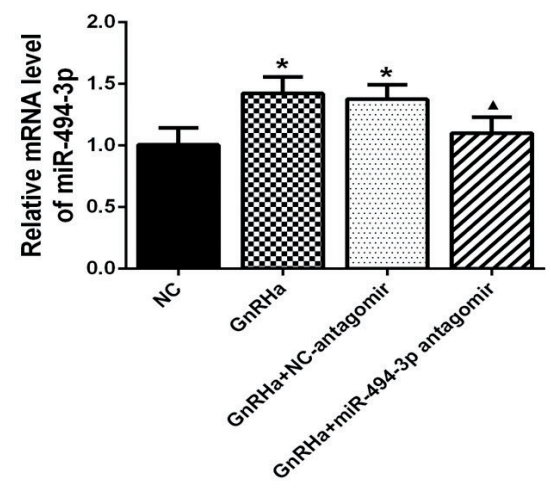

C

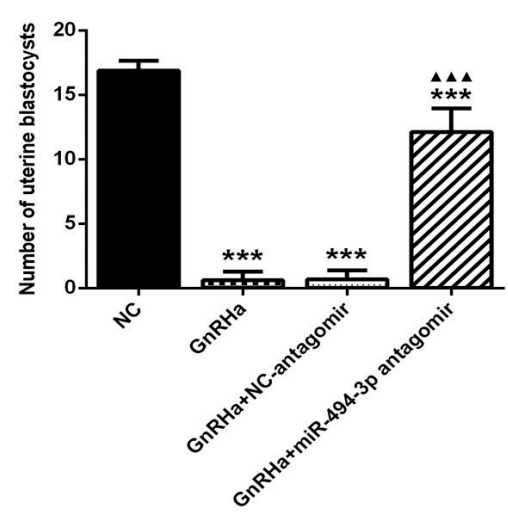

B

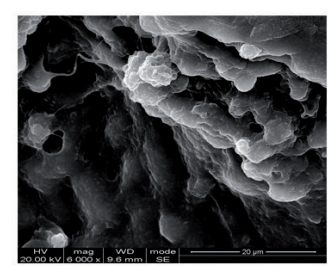

NC

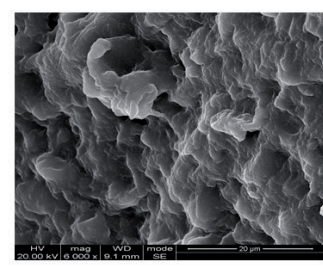

GnRHa+NC-antagomir

D

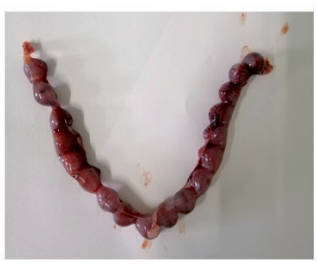

NC

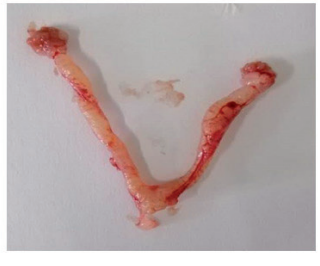

GnRHa+NC-antagomir

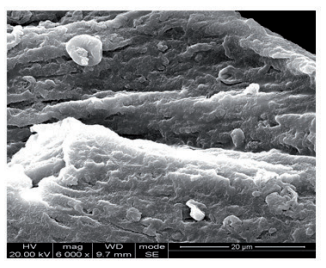

GnRHa

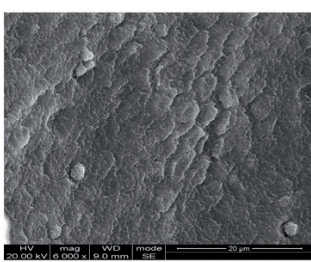

GnRHa+miR-494-3p antagomir

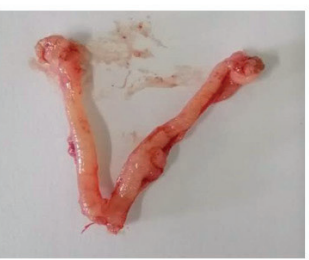

GnRHa

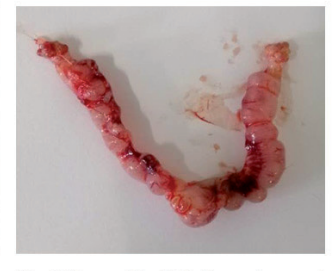

GnRHa+miR-494-3p antagomir
Figure 1. Effect of miR-494-3p antagomir on embryo implantation in mice. A. The expression of miR-494-3p in mouse EECs after injecting miR-494$3 \mathrm{p}$ antagomir, NC-antagomir or RNase-free water. B. The expression of pinopodes, observed by scanning electron microscopy $(6000 \times)$. C. Total number of uterine blastocysts. D. Implantation sites. Data are means $\pm \mathrm{SD}(n=5$ in each group). ${ }^{*} p<0.05,{ }^{* * *} p<$ $0.001 v$ s. NC; $\boldsymbol{\Delta} \boldsymbol{\Delta} \boldsymbol{\Delta} p<0.001$ vs. GnRHa. NC, negative control group; GnRHa, group injected with GnRHa; GnRHa+NCantagomir, group injected with GnRHa and NC-antagomir; GnRHa+miR-494-3p antagomir, group injected with GnRHa and miR-494-3p antagomir. 
podes were inhibited or degraded, and there were few fully developed pinopodes, accompanied by long endometrial surface microvilli. Among the rare microvilli, 1-2 immature pinopodes with different size and irregular shape were seen occasionally, and their protuberances were not obvious. In the GnRHa+miR494-3p-antagomir group, several developing pinopodes could be seen on the endometrial surface, and part of the pinopodes had regular surface morphology with clear boundaries (Fig. 1B).

The blastocysts implanted in the endometrium were counted and the implantation sites in each group were observed. Compared with the NC group, the total number of uterine blastocysts was significantly decreased in both the GnRHa group and the GnRHa+NC-antagomir group. Compared with the GnRHa group, the total number of uterine blastocysts was significantly increased in the GnRHa+miR494-3p-antagomir group (Fig. 1C). The implantation sites presented in the NC group were obviously visible, while only a few immature implantation sites were occasionally seen in both the GnRHa group and the GnRHa+NC-antagomir group. In addition, in the $\mathrm{GnRHa}+\mathrm{miR}-494-3 \mathrm{p}$-antagomir group, many relatively clear implantation sites were observed (Fig. 1D).

\section{Inhibition of miR-494-3p increased endometrial thickness}

To investigate the effect of miR-494-3p on endometrial thickness, HE staining was used to observe the endometrial thick- ness of mice in each group (Fig. 2A). The statistical results show that, compared with the NC group, the endometrial thickness of mice in the GnRHa group and the GnRHa+NCantagomir group was significantly reduced. Compared with the GnRHa group, the endometrial thickness of mice in the GnRHa+miR-494-3p-antagomir group was significantly increased (Fig. 2B).

Inhibition of miR-494-3p increased the protein expressions of LIF, Ang-2 and VEGF

Western blotting assay results showed that, compared with the NC group, the protein expression levels of LIF, Ang-2 and VEGF in the GnRHa group and the GnRHa+NC-antagomir group was significantly reduced. Of note, compared with the GnRHa group, the protein levels of these three factors in the GnRHa+miR-494-3p-antagomir group were significantly upregulated (Fig. 3A,B).

\section{Inhibition of miR-494-3p regulated the PI3K/AKT pathway}

To investigate the effect of miR-494-3p on PI3K/AKT pathway, Western blotting assay was used to observe the relative protein expressions of AKT, p-AKT, mTOR and p-mTOR in endometrial tissues. The results showed that, compared with the NC group, the relative protein expressions of p-AKT (p-AKT/AKT) and p-mTOR (p-mTOR/mTOR) in the GnRHa group and the GnRHa+NC-antagomir group all
A

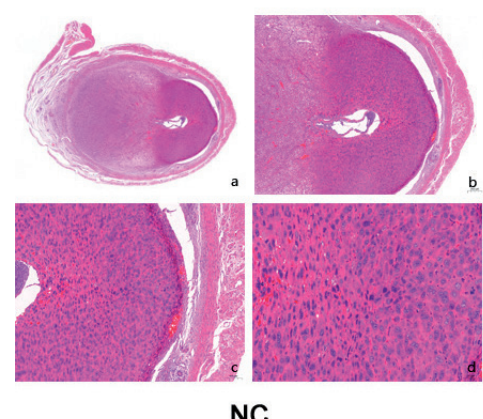

NC

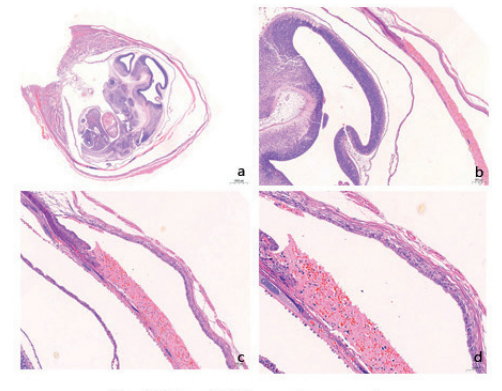

GnRHa+NC-antagomir

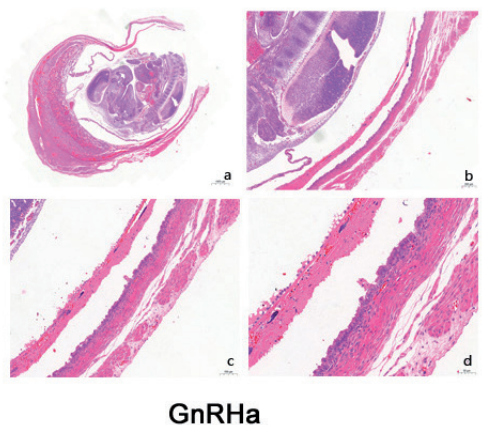

GnRHa

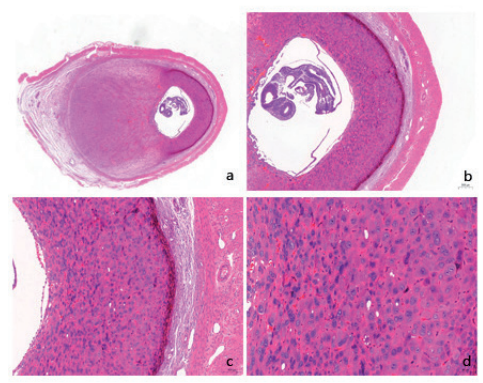

GnRHa+miR-494-3p antagomir
B

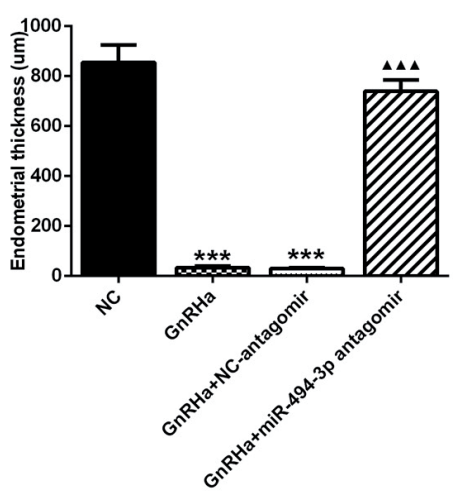

Figure 2. Effect of miR-494-3p antagomir on endometrial thickness in mice. A. Endometrial thickness was observed under a biological microscope using HE staining. (a, b, c, d stands for $10 \times, 40 \times, 100 \times$ and $200 \times$, respectively). B. Bar plot showing summary of results in each group. Data are means $\pm \mathrm{SD}(n=5$ in each group). ${ }^{* *} p<0.001 v s$. NC; ${ }^{\star \Delta \wedge} p<0.001 v s$. GnRHa. For abbreviations, see Fig. 1. 
A

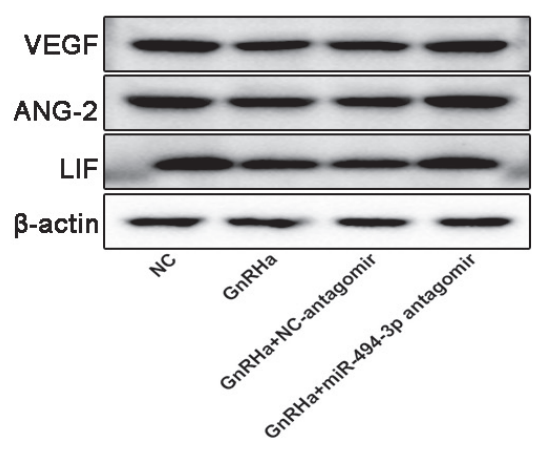

B

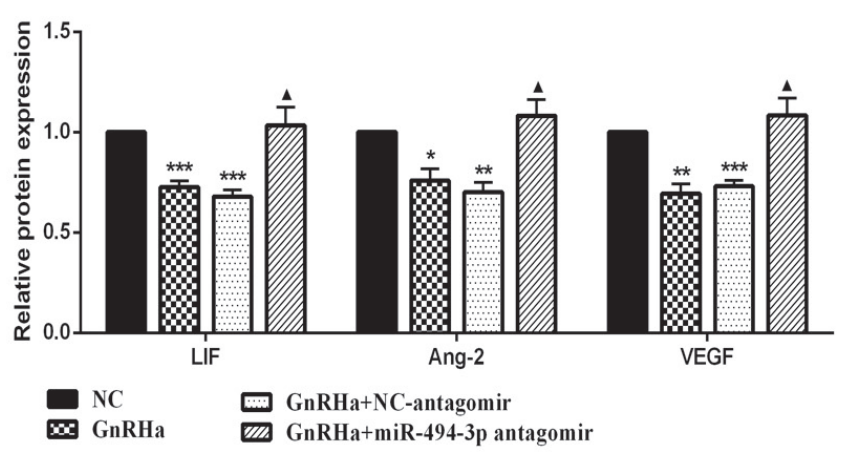

Figure 3. Effect of miR-494-3p antagomir on LIF, Ang-2 and VEGF of endometrial tissues in mice. Protein bands (A) and relative protein expressions (B) of LIF, Ang-2 and VEGF in endometrial tissues by Western blotting assay in each group. Expression was normalized to $\beta$-actin. Data are means $\pm \mathrm{SD}(n=5$ in each group $) .{ }^{\star} p<0.05,{ }^{* *} p<0.01,{ }^{* *} p<0.001 v s$. NC; ${ }^{\star} p<0.05 v s$. GnRHa. ANG-2, Angiopoietin-2; LIF, leukemia inhibitory factor; VEGF, vascular endothelial growth factor. For more abbreviations, see Fig. 1.

showed a significant downregulation trend, whereas AKT and mTOR did not show significant difference. However, compared with the GnRHa group, the protein levels of $\mathrm{p}$ AKT (p-AKT/AKT) and p-mTOR (p-mTOR/mTOR) in the GnRHa+miR-494-3p-antagomir group showed a significant upregulation trend, similarly, AKT and mTOR did not show significant difference (Fig. 4A-C).

\section{Prediction and confirmation of the miR-494-3p target genes}

To predict the target gene of miR-494-3p, we used 10 online target prediction programs (DIANAmT, miRanda, miRDB, miRWalk, RNAhybrid, PICTAR4, PICTAR5, PITA, RNA22, Targetscan): LIF was one of the target genes predicted by five programs (Fig. 5A).
To verify the putative target gene, we performed the dual-luciferase reporter assay. The 3'UTR fragment of LIF containing the binding site was cloned immediately downstream of the firefly luciferase reporter gene into pcDNA vector (LIF-3'UTR-wt). LIF-3'UTR-wt reporter vector was respectively co-transfected with NC mimic or miR-494-3p mimic in hEECs. The luciferase activity in the group of cotransfection with miR-494-3p mimic and LIF-3'UTR-wt led to a significant decrease $(p<0.001)$ compared with the NC-mimic group (Fig. 5B). These results suggest that miR494-3p may regulate gene expression by binding to the seed sequence in the 3'UTR region of LIF.

To further investigate whether LIF is the target gene of miR-494-3p, the expression of LIF protein was examined in hEECs after transfection with miR-494-3p inhibitor.
A

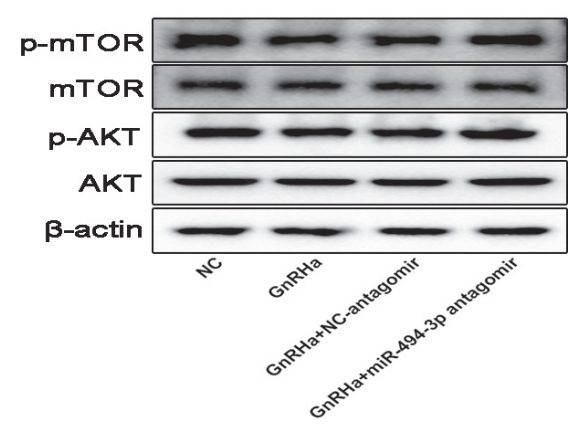

B

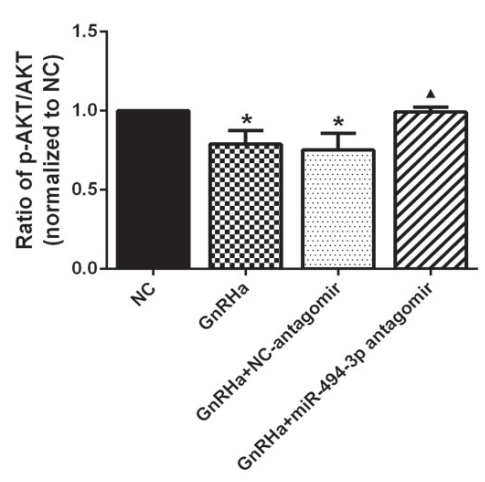

C

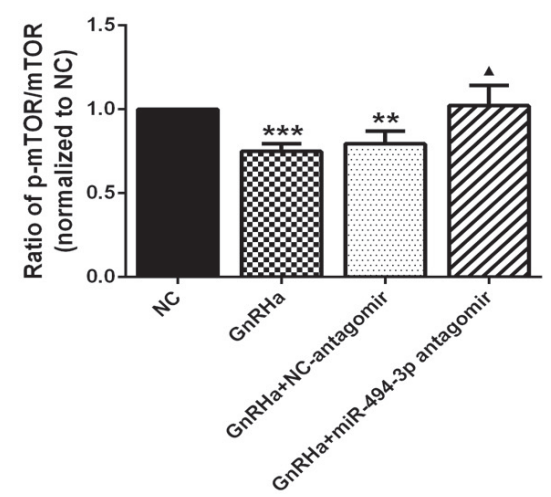

Figure 4. Effect of miR-494-3p antagomir on PI3K/AKT pathway of endometrial tissues in mice. A. Protein bands for AKT, p-AKT, mTOR and $\mathrm{p}$-mTOR by Western blotting assay. Relative protein expressions of p-AKT/AKT (B) and p-mTOR/mTOR (C). Expression was normalized to $\beta$-actin. Data are means $\pm \operatorname{SD}(n=5$ in each group $){ }^{*} p<0.05,{ }^{* *} p<0.01,{ }^{* * *} p<0.001 v s$. NC; ${ }^{\star} p<0.05 v s$. GnRHa. For abbreviations, see Fig. 1. 
A

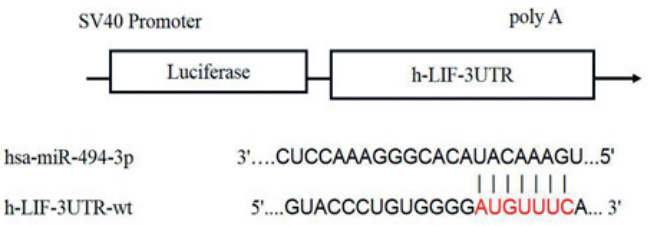

C

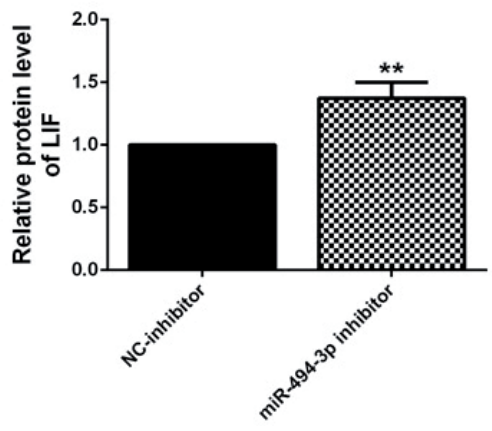

B

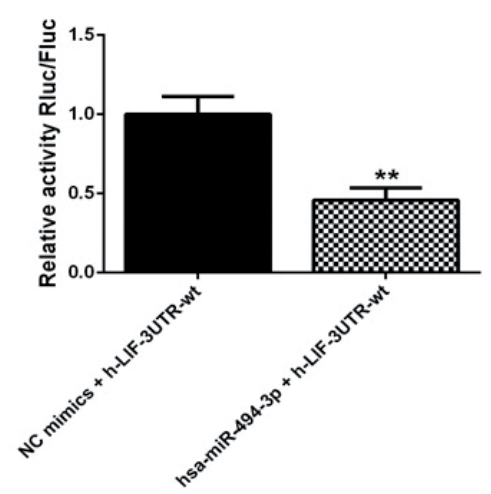

D

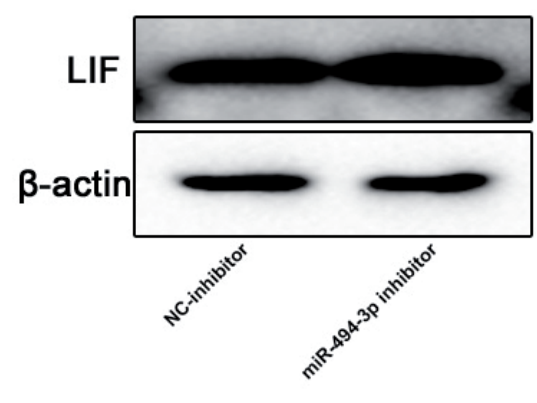

Figure 5. Prediction and confirmation of the miR-494-3p target gene. A. Binding sites between miR-494$3 p$ and LIF-3'UTR-wt. B. Relative luciferase activity (LIF-3'UTR-wt) in miR-494-3p mimic and NC mimic transfected hEECs. ${ }^{* *} p<0.01 v s$. NC mimics group. Western blotting assay (C) and relative protein expression (D) of LIF in the NC inhibitor or miR-494-3p transfected hEECs. Data are means $\pm \mathrm{SD}$ ( $n=3$ in each group). ${ }^{* *} p<0.01$ vs. NC-inhibitor group. For abbreviations, see Fig. 1.
miR-494-3p inhibitor caused significant upregulation of LIF protein expression (Fig. 5C,D)

\section{miR-494-3p regulates cell proliferation, invasiveness and apoptosis}

The establishment of endometrial receptivity involves the change of intracellular environment, including apoptosis and proliferation. The balance of endometrial cell apoptosis is very important for the establishment of peri-implantation uterine function, and the decrease of endometrial receptivity is related to the abnormal decrease of endometrial cell apoptosis. To investigate whether miR-494-3p inhibitor affects the biological function of hEEC, RT-qPCR was firstly used to detect the expression of miR-494-3p in hEECs of each group for the potency testing, and the results showed that miR-494-3p inhibitor did significantly inhibit the expression of miR-494-3p (Fig. 6A). Then CCK-8 assay, transwell assay and flow cytometry were performed. The results showed that, miR494-3p inhibitor significantly increased the proliferation and invasion and significantly inhibited the apoptosis in hEECs. In addition, when miR-494-3p inhibitor and LY294002 (PI3K inhibitor) were co-transferred into hEECs, cell proliferation and invasion were significantly decreased and apoptosis was significantly increased in the miR-494-3p inhibitor+LY294002 group compared with the miR-494-3p inhibitor group (Fig. 6B-F). This suggested that LY294002, as a PI3K inhibitor, reversed the biological function of miR-494-3p inhibitor for hEECs, which provided a preliminary regulation for the subsequent analysis of the regulatory effect of miR-494-3p on PI3K/ AKT pathway in hEECs.

\section{miR-494-3p regulates PI3K/AKT pathway in hEECs}

To investigate the effect of miR-494-3p inhibitor on PI3K/ AKT pathway in hEECs, relative protein expressions of AKT, p-AKT, mTOR and p-mTOR were observed by Western blotting assay in each group. The results showed that, compared with the NC group, the relative protein expressions of p-AKT (p-AKT/AKT) and p-mTOR (p-mTOR/ mTOR) in hEECs were significantly increased in the miR-494-3p inhibitor group, with no significance of AKT and mTOR expressions (Fig. 7A-C). While the relative protein expressions of $\mathrm{p}-\mathrm{AKT} / \mathrm{AKT}$ and $\mathrm{p}-\mathrm{mTOR} / \mathrm{mTOR}$ in hEECs were significantly decreased in the miR-494-3p inhibitor+LY294002 group compared with the miR-494-3p inhibitor group, with no significance of AKT and mTOR expressions (Fig. 7A-C). 
A

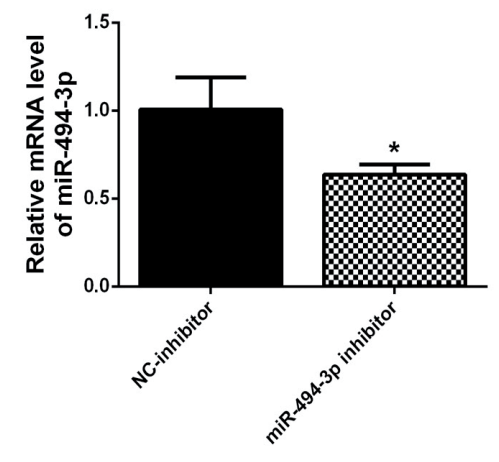

B

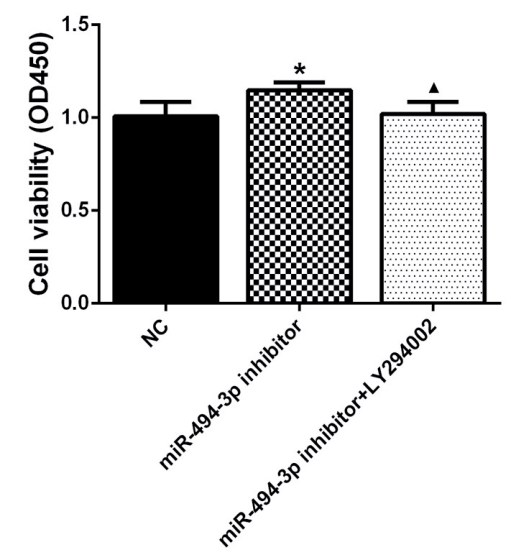

C

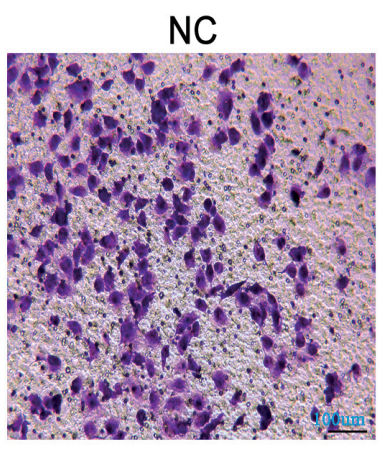

miR-494-3p inhibitor

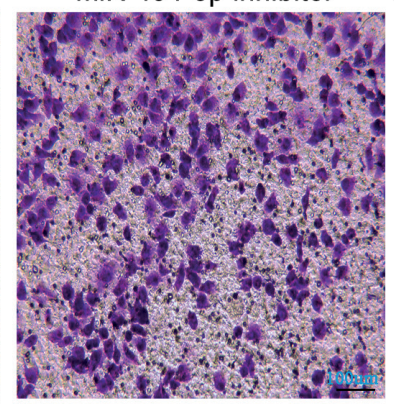

D

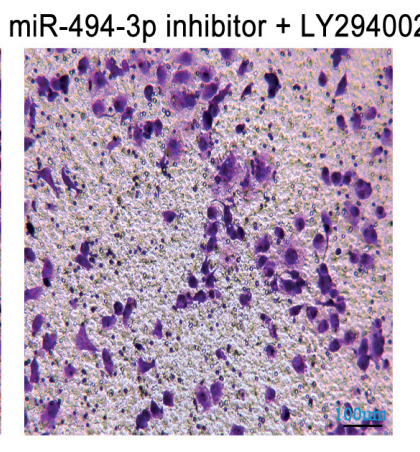

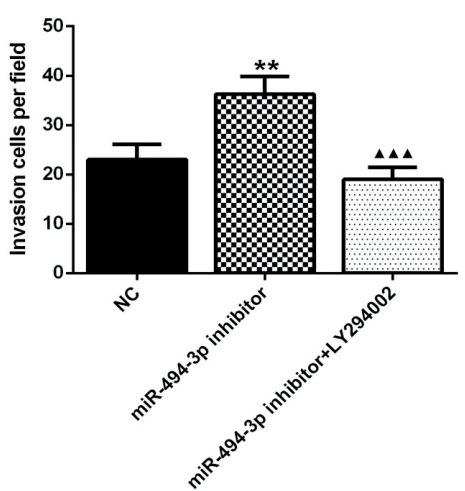

$\mathbf{F}$
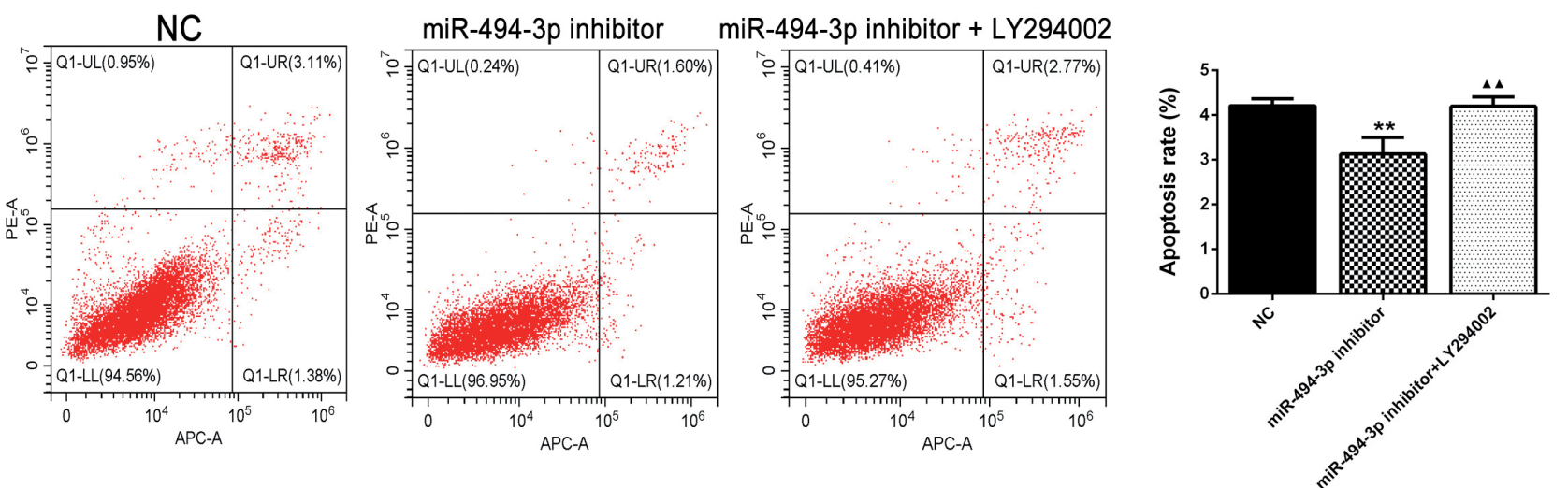

Figure 6. Effect of miR-494-3p inhibitor on biological behavior in human uterine epithelial cells (hEECs). A. The expression of miR494-3p in the NC inhibitor or miR-494-3p inhibitor transfected hEECs. Data are means \pm SD $\left(n=3\right.$ in each group). ${ }^{*} p<0.05 v s$. NCinhibitor group. B. CCK8 was performed to detect the viability of hEECs in each group. Data are means \pm SD ( $n=3$ in each group). ${ }^{*} p<0.05 v s$. NC group, $\wedge p<0.05 v s$. miR-494-3p inhibitor group. C., D. Transwell assay was performed to detect the invasion of hEECs in each group. Data are means $\pm \mathrm{SD}\left(n=3\right.$ in each group). ${ }^{\star *} p<0.01 v s$. NC group, $\Delta \Delta \wedge<0.001 v s$. miR-494-3p inhibitor group. E., F. Flow cytometry was performed to detect the apoptosis of hEECs in each group. Data are means $\pm \mathrm{SD}$ ( $n=3$ in each group). ${ }^{* *} p<$ $0.01 v s$. NC group, ${ }^{\wedge} \boldsymbol{p} p<0.01$ vs. miR-494-3p inhibitor group. For abbreviations, see Fig. 1. 
A

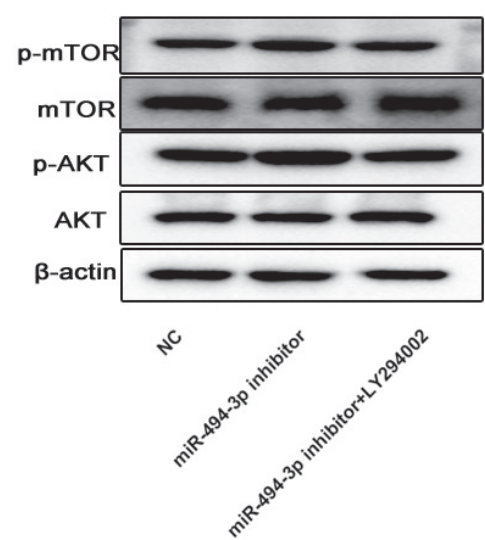

B

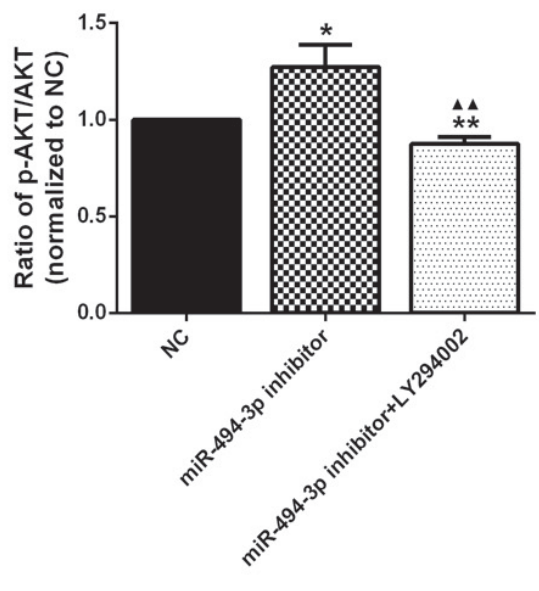

C

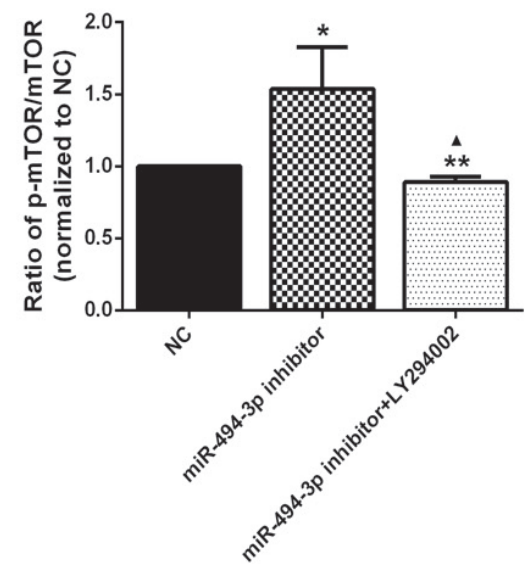

Figure 7. Effect of miR-494-3p inhibitor on PI3K/AKT pathway in hEECs. A. Protein bands for AKT, p-AKT, mTOR and p-mTOR by Western blotting assay. Relative protein expressions of p-AKT/AKT (B) and p-mTOR/mTOR $(\mathbf{C})$ in hEECs by Western blotting assay in each group. Expression was normalized to $\beta$-actin. Data are means $\pm \operatorname{SD}\left(n=3\right.$ in each group). ${ }^{\star} p<0.05,{ }^{* *} p<0.01 v s$. NC group; $\Delta p<0.05, \Delta \wedge<0.01$ vs. miR-494-3p inhibitor group. For abbreviations, see Fig. 1.

\section{Discussion}

In this study, we constructed GnRHa superovulation mice to explore the mechanism of miR-494-3p on endometrial receptivity in superovulation mice, and confirmed that LIF was a potential target of miR-494-3p. In terms of cell function, miR-494-3p inhibited hEEC cell viability and metastasis. Importantly, miR-494-3p was shown to block the PI3K/AKT pathway and induce apoptosis by regulating LIF expression. These results suggest that miR-494-3p plays an important role in the regulation of endometrial receptivity. Data have shown that ovulation stimulation treatment has a negative effect on endometrial function and miRNA expression. For example, superovulation in mice and humans altered the expression of miR-200, thus negatively affecting endometrial development and decidua formation (Jimenez et al. 2016). The dosage of GnRHa in this experiment resulted in endometrial damage and abnormal LIF expression in the rat model, which ultimately affected the endometrial implantation ability in the later stage. Predictably, the GnRHa superovulation regimen had been shown to damage the endometrium and implant at a very early stage, and our model building methods and observations were consistent with the previously confirmed results (Gong et al. 2013). Our findings suggested that hormonal changes associated with superovulation may adversely affect endometrial receptivity by inducing abnormal miR-494-3p expression.

Most scholars believed that the appearance of pinocytosis in endometrial histomorphology was the gold standard of endometrial receptivity. The pinopode was the membrane protrusion on the top of the membrane of the endome- trium epithelium during the implantation window under scanning electron microscope. Fully developed pinocytosis was an important morphological indicator of endometrial receptivity establishment and implantation window opening (Quinn et al. 2020). In this study, GnRHa, HMG and HCG were intraperitoneally injected into mice during preimplantation period to create a model of blastocyst implantation disorder. Our results showed that miR-494-3p antagomir increased developing pinopodes and endometrial thickness of mice, indicating that miR-494-3p antagomir improved endometrial receptivity.

The process of embryo implantation in successful pregnancy involves the adhesion of trophoblastic layers to the receptive endometrium. Significant changes in the expression of adhesion molecules in endometrium have an effect on endometrial receptivity. LIF plays a role in the adhesion and infiltration stages of implantation (Dimitriadis et al. 2010). Moreover, LIF was a key factor in the regulation of embryo implantation (Schmitz et al. 2017). VEGF receptor 2 (VEGFR-2) was considered to be the most important VEGF receptor during angiogenesis and was known to regulate endometrial angiogenesis (Waltenberger et al. 1994). VEGF was highly expressed in endometrium in the middle stage of secretion. It is an important factor in promoting endometrial angiogenesis (Raine-Fenning et al. 2008). Besides, Ang-2 was involved in villi formation during implantation and the establishment of fetal and maternal capillary network, which was very important for maintaining pregnancy (Fiorimanti et al. 2018). It suggests that LIF, VEGF and Ang-2 play key roles in endometrial receptivity. In this study, we found that inhibition of miR-494-3p up-regulated the expressions of 
LIF, Ang-2 and VEGF, thus improving embryo implantation (Schmitz et al. 2017) and maintaining pregnancy (Fiorimanti et al. 2018) to improve endometrial receptivity and promote embryo implantation (Segal et al. 2020). Interestingly, VEGF attenuated the HIF-1a signaling reducing resistance to antiangiogenic therapy (Tsai et al. 2016). These results suggest that there is an association between VEGF/HIF and inhibition of miR-494-3p in improving endometrial receptivity recovery. Together these results provide important insights into improvement of endometrial receptivity that inhibition of miR-494-3p improved endometrial proliferation and adhesion functions by promoting the expression of endometrial LIF, Ang-2 and VEGF during implantation, and significantly improve tissue endometrial receptivity. Extracellular matrix degradation is the basic process that initiates blastocystcyst invasion (Maia-Filho et al. 2015). The establishment of tolerance endometrium is a complex and dynamic process, during which the morphology and physiological state of endometrium undergo a series of significant changes, including cell proliferation and apoptosis (Chen et al. 2020). Therefore, it is necessary to continue to explore the regulatory role of miR-494-3p on hEECs. Importantly, our results showed that miR-494-3p inhibitor promoted hEECs proliferation and inhibited hEECs apoptosis, suggesting that miR-494$3 p$ inhibitor enhanced hEEC cell viability and metastasis.

The PI3K/AKT/mTOR signaling pathway downstream of VEGFR-2 has been shown to modulate angiogenesis in endometriosis models through a series of cascade responses (Cao et al. 2017). It is well known that PI3K/AKT/mTOR is a key activation signaling pathway regulating cell proliferation, apoptosis and survival (Mitra et al. 2012; Pan et al. 2015). $\mathrm{PI} 3 \mathrm{~K} / \mathrm{AKT}$ pathway plays an important role in the process of decidualization process of endometrial stromal cells (Fabi et al. 2017). Studies in recent years have shown that VEGFA can participate in cell proliferation and differentiation by activating its downstream PI3K/AKT/mTOR signaling pathway (Chen et al. 2014; Sadremomtaz et al. 2018). What emerges from the results reported here was that the inhibition of miR-494-3p activated PI3K/AKT/mTOR pathway by up-regulating VEGF/HIF signal, thereby promoting cell proliferation and invasion and inhibiting cell apoptosis. These results corroborated the findings of a great deal of the previous work that activation of the PI3K/AKT/mTOR pathway increased the secretion of VEGF through HIF-1 dependence and independent mechanism, which is crucial for normal vascular development during embryogenesis (Karar et al. 2011), thus improving endometrial receptivity.

\section{Conclusion}

In conclusion, our study supported that miR-494-3p targeting LIF regulated endometrial receptivity via the PI3K/AKT/
mTOR pathway. Inhibition of miR-494-3p had the potential to improve the treatment of female infertility associated with reduced endometrial receptivity. However, further studies on the specific regulatory mechanisms of miR-494-3p in endometrial receptivity will be the goal of our future studies.

Acknowledgements. This study was supported by the Xinglin Scholar Research Premotion Project of Chengdu University of TCM (grant No. 2019yky08).

Availability of data and materials. The datasets used during the present study are available from the corresponding author on reasonable request.

Authors' contributions. LY and FF contributed to conception and design of the study. LY and RXJ contributed to acquisition, analysis and interpretation of the data and writing the manuscript. $\mathrm{ZM}$ and JZH, YL and YLL discussed the results and implications and commented on the manuscript at all stages, as well as in the final approval of the version to be published.

Ethics approval and consent to participate. All the experimental procedures were approved by and performed in accordance with the West China Hospital of Sichuan University (Chengdu, China).

Conflict of interest. The authors declare that they have no competing interests.

\section{References}

Altmäe S, Martinez-Conejero JA, Esteban FJ, Ruiz-Alonso M, Stavreus-Evers A, Hocajadas JA, Salumets A (2013): MicroRNAs miR-30b, miR-30d, and miR-494 regulate human endometrial receptivity. Reprod. Sci. 20, 308-317 https://doi.org/10.1177/1933719112453507

Cao Y, Ye Q, Zhuang M, Xie S, Zhong R, Cui J, Zhou J, Zhu Y, Zhang T, Cao L (2017): Ginsenoside Rg3 inhibits angiogenesis in a rat model of endometriosis through the VEGFR-2-mediated PI3K/ Akt/mTOR signaling pathway. PloS One 12, e0186520 https://doi.org/10.1371/journal.pone.0186520

Chen B, Zhang C, Dong P, Guo Y, Mu N (2014): Molecular regulation of cervical cancer growth and invasion by VEGFa. Tumour Biol. 35, 11587-11593 https://doi.org/10.1007/s13277-014-2463-2

Chen Q, Ni Y, Han M, Zhou WJ, Zhu XB, Zhang AJ (2020): Integrinlinked kinase improves uterine receptivity formation by activating Wnt $/ \beta$-catenin signaling and up-regulating MMP-3/9 expression. Am. J. Transl. Res. 12, 3011-3022

Chen T, Dong J, Zhou H, Deng X, Li R, Chen N, Luo M, Li Y, Wu J, Wang L (2020): Glycation of fibronectin inhibits VEGF-induced angiogenesis by uncoupling VEGF receptor-2-c-Src crosstalk. J. Cell. Mol. Med. 24, 9154-9164 https://doi.org/10.1111/jcmm.15552

Chuffa LGA, Lupi LA, Cucielo MS, Silveira HS, Reiter RJ, Ferreira Seiva FR (2019): Melatonin promotes uterine and placental health: potential molecular mechanisms. Int. J. Mol. Sci. 21, 300 
https://doi.org/10.3390/ijms21010300

Dimitriadis E, Nie G, Hannan NJ, Paiva P, Salamonsen LA (2010): Local regulation of implantation at the human fetal-maternal interface. Int. J. Dev. Biol. 54, 313-322 https://doi.org/10.1387/ijdb.082772ed

Fabi F, Grenier K, Parent S, Adam P, Tardif L, Leblanc V, Asselin E (2017): Regulation of the PI3K/Akt pathway during decidualization of endometrial stromal cells. PloS One 12, e0177387 https://doi.org/10.1371/journal.pone.0177387

Fiorimanti MR, Rabaglino MB, Cristofolini AL, Merkis CI (2018): Immunohistochemical determination of Ang-1, Ang-2 and Tie-2 in placentas of sows at 30, 60 and 114 days of gestation and validation through a bioinformatic approach. Anim. Reprod. Sci. 195, 242-250 https://doi.org/10.1016/j.anireprosci.2018.06.001

Franasiak JM, Holoch KJ, Yuan L, Schammel DP, Young SL, Lessey BA (2014): Prospective assessment of midsecretory endometrial leukemia inhibitor factor expression versus $\alpha v \beta 3$ testing in women with unexplained infertility. Fertil. Steril. 101, 1724-1731 https://doi.org/10.1016/j.fertnstert.2014.02.027

Gong X, Yu Y, Tong Q, Ren Y, Jin Z (2013): Effects of „Bu Shen Huo Xue Decoction“ on the endometrial morphology and expression of leukaemia inhibitory factor in the rat uterus during the oestrous cycle. Evid. Based Complement Alternat. Med. 2013, 496036 https://doi.org/10.1155/2013/496036

Griffiths-Jones S, Grocock RJ, van Dongen S, Bateman A, Enright AJ (2006): miRBase: microRNA sequences, targets and gene nomenclature. Nucleic Acids Res. 34, D140-144 https://doi.org/10.1093/nar/gkj112

Guo F, Si C, Zhou M, Wang J, Zhang D, Leung PCK, Xu B, Zhang A (2018): Decreased PECAM1-mediated TGF- $\beta 1$ expression in the mid-secretory endometrium in women with recurrent implantation failure. Hum. Reprod. 33, 832-843 https://doi.org/10.1093/humrep/dey022

Hosseini S, Hosseini S, Salehi M (2020): Upregulation of Toll-like receptor 4 through anti-miR-Let-7a enhances blastocyst attachment to endometrial cells in mice. J. Cell. Physiol. 235, 9752-9762 https://doi.org/10.1002/jcp.29787

Huang W, Liang Y, Chung HY, Wang G, Huang JJ, Li Y (2020): Cyperenoic acid, a sesquiterpene derivative from Croton crassifolius, inhibits tumor growth through anti-angiogenesis by attenuating VEGFR2 signal pathway in breast cancer. Phytomedicine $\mathbf{7 6}, 153253$

https://doi.org/10.1016/j.phymed.2020.153253

Jimenez PT, Mainigi MA, Word RA, Kraus WL, Mendelson CR (2016): miR-200 regulates endometrial development during early pregnancy. Mol. Endocrinol. 30, 977-987 https://doi.org/10.1210/me.2016-1050

Kaczmarek MM, Blitek A, Schams D, Ziecik AJ (2010): Effect of luteinizing hormone and tumour necrosis factor-alpha on VEGF secretion by cultured porcine endometrial stromal cells. Reprod. Domest. Anim. 45, 481-486 https://doi.org/10.1111/j.1439-0531.2008.01266.x

Karar J, Maity A (2011): PI3K/AKT/mTOR pathway in angiogenesis. Front. Mol. Neurosci. 4, 51 https://doi.org/10.3389/fnmol.2011.00051

Kim JO, Ahn EH, Sakong JH, An HJ, Park HS, Kim YR, Lee JR, Lee WS, Kim NK (2020): Association of miR-27aA $>$ G, miR-423C >a, miR-449bA $>$ G, and miR-604A $>$ G polymorphisms with risk of recurrent implantation failure. Reprod. Sci. 27, 29-38 https://doi.org/10.1007/s43032-019-00031-6

Kunicka Z, Kurzyńska A, Szydłowska A, Bogacka I (2020): Peroxisome proliferator-activated receptor alpha regulates the expression of the immune response mediators in the porcine endometrium during the estrous cycle and early pregnancy. Am. J. Reprod. Immunol. 83, e13211 https://doi.org/10.1111/aji.13211

Li H, Zhao B, Liu Y, Deng W, Zhang Y (2020): Angiogenesis in residual cancer and roles of HIF-1 $\alpha$, VEGF, and MMP-9 in the development of residual cancer after radiofrequency ablation and surgical resection in rabbits with liver cancer. Folia Morphol. (Warsz.) 79, 71-78

Maia-Filho VO, Rocha AM, Ferreira FP, Bonetti TC, Serafini P, Motta EL (2015): Matrix metalloproteinases 2 and 9 and e-cadherin expression in the endometrium during the implantation window of infertile women before in vitro fertilization treatment. Reprod. Sci. 22, 416-422 https://doi.org/10.1177/1933719114529373

Mitra A, Raychaudhuri SK, Raychaudhuri SP (2012): IL-22 induced cell proliferation is regulated by $\mathrm{PI} 3 \mathrm{~K} / \mathrm{Akt} / \mathrm{mTOR}$ signaling cascade. Cytokine 60, 38-42 https://doi.org/10.1016/j.cyto.2012.06.316

Mahajan N (2015): Endometrial receptivity array: Clinical application. J. Hum. Reprod. Sci. 8, 121-129 https://doi.org/10.4103/0974-1208.165153

Nikas G, Develioglu OH, Toner JP, Jones HW (1999): Endometrial pinopodes indicate a shift in the window of receptivity in IVF cycles. Hum. Reprod. 14, 787-792 https://doi.org/10.1093/humrep/14.3.787

Pan ST, Qin Y, Zhou ZW, He ZX, Zhang X, Yang T, Yang YX, Wang D, Qiu JX, Zhou SF (2015): Plumbagin induces G2/M arrest, apoptosis, and autophagy via p38 MAPK- and PI3K/Akt/ mTOR-mediated pathways in human tongue squamous cell carcinoma cells. Drug Des. Devel. Ther. 9, 1601-1626 https://doi.org/10.2147/DDDT.S76057

Quinn KE, Matson BC, Wetendorf M, Caron KM (2020): Pinopodes: Recent advancements, current perspectives, and future directions. Mol. Cell. Endocrinol. 501, 110644

https://doi.org/10.1016/j.mce.2019.110644

Raine-Fenning N (2008): Doppler assessment of uterine artery blood flow for the prediction of pregnancy after assisted reproduction treatment. Ultrasound Obstet. Gynecol. 31, 371-375 https://doi.org/10.1002/uog.5321

Sadremomtaz A, Mansouri K, Alemzadeh G, Safa M, Rastaghi AE, Asghari SM (2018): Dual blockade of VEGFR1 and VEGFR2 by a novel peptide abrogates VEGF-driven angiogenesis, tumor growth, and metastasis through PI3K/AKT and MAPK/ERK1/2 pathway. Biochim. Biophys. Acta 1862, 2688-2700 https://doi.org/10.1016/j.bbagen.2018.08.013

Schmitz CR, Oehninger S, Genro VK, Chandra N, Lattanzio F, Yu L, Cunha-Filho JS (2017): Alterations in expression of 
endometrial milk fat globule-EGF factor 8 (MFG-E8) and leukemia inhibitory factor (LIF) in patients with infertility and endometriosis. JBRA Assist. Reprod. 21, 313-320 https://doi.org/10.5935/1518-0557.20170056

Segal TR, Amini P, Wang J, Peters G, Skomorovska-Prokvolit Y, Mainigi MA, Goldfarb JM, Mesiano S, Weinerman R (2020): Superovulation with human chorionic gonadotropin (hCG) trigger and gonadotropin releasing hormone agonist (Gn$\mathrm{RHa}$ ) trigger differentially alter essential angiogenic factors in the endometrium in a mouse ART model. Biol. Reprod. 102, $1122-1133$ https://doi.org/10.1093/biolre/ioaa014

Tsai JL, Lee YM, Pan CY (2016): The novel VEGF121-VEGF165 fusion attenuates angiogenesis and drug resistance via targeting VEGFR2-HIF-1 $\alpha$-VEGF165/lon signaling through PI3KAKT-mTOR pathway. Curr. Cancer Drug Targets 16, 275-286 https://doi.org/10.2174/156800961603160206125352

Waltenberger J, Claesson-Welsh L, Siegbahn A, Shibuya M, Heldin CH (1994): Different signal transduction properties of KDR and Flt1, two receptors for vascular endothelial growth factor. J. Biol. Chem. 269, 26988-26995

https://doi.org/10.1016/S0021-9258(18)47116-5
Xu X, Li Z, Liu J, Yu S, Wei Z (2017): MicroRNA expression profiling in endometriosis-associated infertility and its relationship with endometrial receptivity evaluated by ultrasound. J. Xray Sci. Technol. 25, 523-532 https://doi.org/10.3233/XST-17286

Yuan L, Feng F, Mao Z, Huang J, Liu Y, Li Y, Jiang R (2020): Effects of Erbuzhuyu decoction combined with acupuncture on endometrial receptivity are associated with the expression of miR494-3p. Evid. Based Complement Alternat. Med. 2020, 9739672 https://doi.org/10.1155/2020/9739672

Zhang L, Liu X, Cui J, Che S, Liu Y, An X, Cao B, Song Y (2019): LncRNA882 regulates leukemia inhibitory factor (LIF) by sponging miR-15b in the endometrial epithelium cells of dairy goat. J. Cell. Physiol. 234, 4754-4767 https://doi.org/10.1002/jcp.27272

Złotkowska A, Adamczyk S, Andronowska A (2019): Presence of trophoblast in the uterine lumen affects VEGF-C expression in porcine endometrium. Theriogenology 125, 216-223 https://doi.org/10.1016/j.theriogenology.2018.11.007

Received: September 25, 2020

Final version accepted: June 29, 2021 